\title{
Performance evaluation for rapid detection of pan-cancer microsatellite instability with MANTIS
}

\author{
Esko A. Kautto ${ }^{1}$, Russell Bonneville ${ }^{1}$, Jharna Miya ${ }^{1}$, Lianbo Yu ${ }^{2}$, Melanie A. Krook ${ }^{1}$, \\ Julie W. Reeser ${ }^{1}$ and Sameek Roychowdhury ${ }^{1,3}$ \\ ${ }^{1}$ Comprehensive Cancer Center, The Ohio State University, Columbus, OH, USA \\ 2 Department of Biomedical Informatics, The Ohio State University, Columbus, $\mathrm{OH}$, USA \\ ${ }^{3}$ Division of Medical Oncology, Department of Internal Medicine, The Ohio State University, Columbus, OH, USA \\ Correspondence to: Sameek Roychowdhury, email: sameek.roychowdhury@osumc.edu \\ Keywords: microsatellite instability, computational biology, next-generation sequencing \\ Received: November 24, $2016 \quad$ Accepted: December 02, $2016 \quad$ Published: December 12, 2016
}

\section{ABSTRACT}

In current clinical practice, microsatellite instability (MSI) and mismatch repair deficiency detection is performed with MSI-PCR and immunohistochemistry. Recent research has produced several computational tools for MSI detection with next-generation sequencing (NGS) data; however a comprehensive analysis of computational methods has not yet been performed. In this study, we introduce a new MSI detection tool, MANTIS, and demonstrate its favorable performance compared to the previously published tools mSINGS and MSISensor. We evaluated 458 normaltumor sample pairs across six cancer subtypes, testing classification performance on variable numbers of target loci ranging from 10 to 2539. All three computational methods were found to be accurate, with MANTIS exhibiting the highest accuracy with $98.91 \%$ of samples from all six diseases classified correctly. MANTIS displayed superior performance among the three tools, having the highest overall sensitivity (MANTIS 97.18\%, MSISensor $96.48 \%$, mSINGS 76.06\%) and specificity (MANTIS 99.68\%, mSINGS 99.68\%, MSISensor $98.73 \%$ ) across six cancer types, even with loci panels of varying size. Additionally, MANTIS also had the lowest resource consumption ( $<1 \%$ of the space and $<7 \%$ of the memory required by mSINGS) and fastest running times (49.6\% and $8.7 \%$ of the running times of MSISensor and mSINGS, respectively). This study highlights the potential utility of MANTIS in classifying samples by MSIstatus, allowing its incorporation into existing NGS pipelines.

\section{INTRODUCTION}

Microsatellites are short (1-6bp) repeating motifs, widely dispersed throughout the human genome [1]. Microsatellite instability (MSI) is a genetic phenomenon of somatic polymorphisms of microsatellite length, caused by uncorrected "slippage" of DNA fragments during DNA replication in cell division. MSI can arise from defects in the DNA mismatch repair (MMR) system [2]. These defects may be inherited, as with Lynch syndrome/hereditary nonpolyposis colorectal cancer [3], or may be somatically acquired, most commonly due to promoter hypermethylation of the MMR gene MLH1 [4]. Increasing evidence demonstrates that MSI is a recurrent somatic abnormality in several human cancers, found in $13 \%$ of colorectal adenocarcinoma and $22-33 \%$ of uterine/ endometrial carcinoma [5]. Reliable detection of MSI is clinically useful as MSI-positive tumors appear more susceptible to immune-enhancing therapies, as observed in colorectal cancer for the PD-1 inhibitor pembrolizumab [6].

The two currently accepted assays for the detection of MSI are MSI-PCR of five standardized microsatellite loci (Bethesda panel) [7], and immunohistochemistry (IHC) of the MMR proteins MSH2, MSH6, MLH1 and PMS2. Traditionally, tumors can be classified with MSIPCR as microsatellite stable (MSS, 0/5 loci unstable), MSI-low (MSI-L, 1/5 loci unstable), or MSI-high (MSI-H, $\geq 2 / 5$ loci unstable) [7]. However, both of these methods have inherent limitations. MSI-PCR relies on a small set of loci that were selected based on markers from a single disease type, potentially excluding loci that would 
Table 1: Comparison of the MSI-calling tools mSINGS, MSISensor and MANTIS, and the algorithms used by each.

\begin{tabular}{|l|l|l|l|}
\hline Tool & Sample Comparison & Statistical Method & Scoring Approach \\
\hline mSINGS & Tumor vs. Baseline & Z-score & Per Locus \\
\hline MSISensor & Tumor vs. Normal & Chi-square & Per Locus \\
\hline MANTIS & Tumor vs. Normal & Average distance & Aggregate Instability \\
\hline
\end{tabular}

Table 2: Breakdown of target loci used for microsatellite status calling. The count and repeat range of each type is listed.

\begin{tabular}{|l|l|l|l|l|}
\hline Type of Microsatellite & Number of Loci & Min Repeats & Max Repeats & Mean Repeats \\
\hline Monomer & 2436 & 3 & 36 & 15.94 \\
\hline Dimer & 96 & 6 & 18 & 14.86 \\
\hline Trimer & 4 & 3 & 8 & 4.75 \\
\hline Tetramer & 2 & 7 & 8 & 7.5 \\
\hline Pentamer & 1 & 3 & 3 & 3.0 \\
\hline
\end{tabular}

be better predictors in other diseases and increasing the odds of incorrect classification [7]. Immunohistochemistry can be used to detect the expression of mismatch repair proteins, but does not directly look at the microsatellite loci [7]. More recently, with the increasing prevalence of next-generation sequencing (NGS) in cancer biology, several computational methods have been developed using either colorectal or endometrial cancer NGS data to determine MSI status [8, 9].

The development, refinement, and validation of NGS-based computational MSI calling methods have several research and clinical applications. NGS allows for the practical assessment of far more microsatellite loci than MSI-PCR. Importantly, computational MSI analyses can be integrated into existing NGS pipelines for other mutation types such as single nucleotide variation or copy number variation, as well as applied to previously generated NGS data for retrospective analyses. As NGS increases in both cost-effectiveness and prevalence, NGS-based methods may permit identification of MSI status without requiring additional clinical testing or patient sample processing. Lastly, NGS data is becoming increasingly available in tumor types that are not routinely tested for MSI, with potential opportunities to identify microsatellite instability in previously uncharacterized cancers. Thus, there is a need to develop tools with high accuracy in multiple cancer types.

In this study, we introduce a new tool, MANTIS (Microsatellite Analysis for Normal Tumor InStability), for detecting MSI status from NGS data. We compare MANTIS with two currently available tools, mSINGS [8] and MSISensor [9], and test their performance across six different tumor types. We also determine that the number of loci assessed impacts the accuracy of MSI calling methods, and find an optimal number of loci to analyze with each tool for best tool performance.

\section{RESULTS}

\section{Evaluation of tool accuracy for detecting MSI status}

Since mSINGS and MSISensor were each developed on data from single disease cohorts (COAD/READ for mSINGS, UCEC for MSISensor), the two cohorts were used for tool performance comparisons. To account for the possibility of suboptimal recommended cutoff thresholds for each of the three tools, we tested a range of thresholds for each tool across a test cohort consisting of both the COAD/READ and UCEC sample pairs (Supplemental Figure S1). The peak performances of each tool were determined, with MANTIS having 97.1\% accuracy with the default threshold of 0.4, mSINGS reaching $96 \%$ accuracy with a threshold of 0.1 , and MSISensor peaking at $95.4 \%$ accuracy with the threshold of $3.5 \%$. The results indicate that MANTIS demonstrated superior performance over the other tools, even after accounting for the bestcase thresholds of the tools.

Having evaluated the tools with the best-case thresholds, their performances were evaluated with the tool's recommended default cutoff thresholds (MANTIS 0.4, mSINGS 0.2, and MSISensor 3.5\%) (Supplemental Figure S2). MANTIS demonstrated the highest classification accuracy (97.1\%), followed by MSISensor (95.4\%), and mSINGS (83.4\%). MANTIS and MSISensor both exhibited equally high sensitivity (95.4\%). In contrast, although mSINGS was the most specific $(100 \%)$, it demonstrated poor sensitivity (66.7\%), largely due to poor performance over the UCEC cohort $(53.1 \%$ sensitivity). MANTIS also exhibited high specificity (98.9\%), performing better than MSISensor (95.5\%).

To analyze disease-specific differences, results were compared between the COAD/READ and UCEC cohorts (Table 3, Supplemental Figure S3). MANTIS 
Table 3: Comparison of accuracy for MSI-H detection.

\begin{tabular}{|l|l|l|l|l|}
\hline mSINGS & COAD/READ & UCEC & COAD/READ + UCEC & STAD \\
\hline Metric & $84.2 \%$ & $53.1 \%$ & $66.7 \%$ & $92.0 \%$ \\
\hline Sensitivity & $100.0 \%$ & $100.0 \%$ & $100 \%$ & $100.0 \%$ \\
\hline Specificity & $92.1 \%$ & $76.8 \%$ & $83.4 \%$ & $96.0 \%$ \\
\hline Accuracy & COAD/READ & UCEC & COAD/READ + UCEC & STAD \\
\hline MSISensor & $98.0 \%$ & $95.4 \%$ & $98.0 \%$ \\
\hline Metric & $92.1 \%$ & $98.0 \%$ & $95.5 \%$ & $100.0 \%$ \\
\hline Sensitivity & $92.1 \%$ & $98.0 \%$ & $95.4 \%$ & $99.0 \%$ \\
\hline Specificity & $92.1 \%$ & \multicolumn{5}{|l|}{} \\
\hline Accuracy & COAD/READ & UCEC & COAD/READ + UCEC & STAD \\
\hline MANTIS & $100.0 \%$ & $91.8 \%$ & $95.4 \%$ & $100.0 \%$ \\
\hline Metric & $100.0 \%$ & $98.9 \%$ & $100.0 \%$ \\
\hline Sensitivity & $97.4 \%$ & $96.0 \%$ & $97.1 \%$ & $100.0 \%$ \\
\hline Specificity & $98.7 \%$ &
\end{tabular}

The performance of mSINGS, MSISensor, and MANTIS over 275 COAD/READ, UCEC, and STAD sample pairs.

produced more accurate results $(98.7 \%)$ than mSINGS $(92.1 \%)$ and MSISensor $(92.1 \%)$ in COAD/READ, whereas MSISensor was slightly more accurate $(98.0 \%)$ than MANTIS (96.0\%) in UCEC. While all three tools performed well with the COAD/READ data, mSINGS performed poorly with the UCEC data (accuracy $76.8 \%$, sensitivity 53.1\%). The tests showed that MANTIS had the most consistently accurate performance among the test cohort, exhibiting high sensitivity $(95.4 \%)$ and specificity $(98.9 \%)$ among the tested samples.

\section{Consideration of data overfitting and bias}

To further evaluate tool performance and to ensure MANTIS was not overfit to the COAD/READ and UCEC

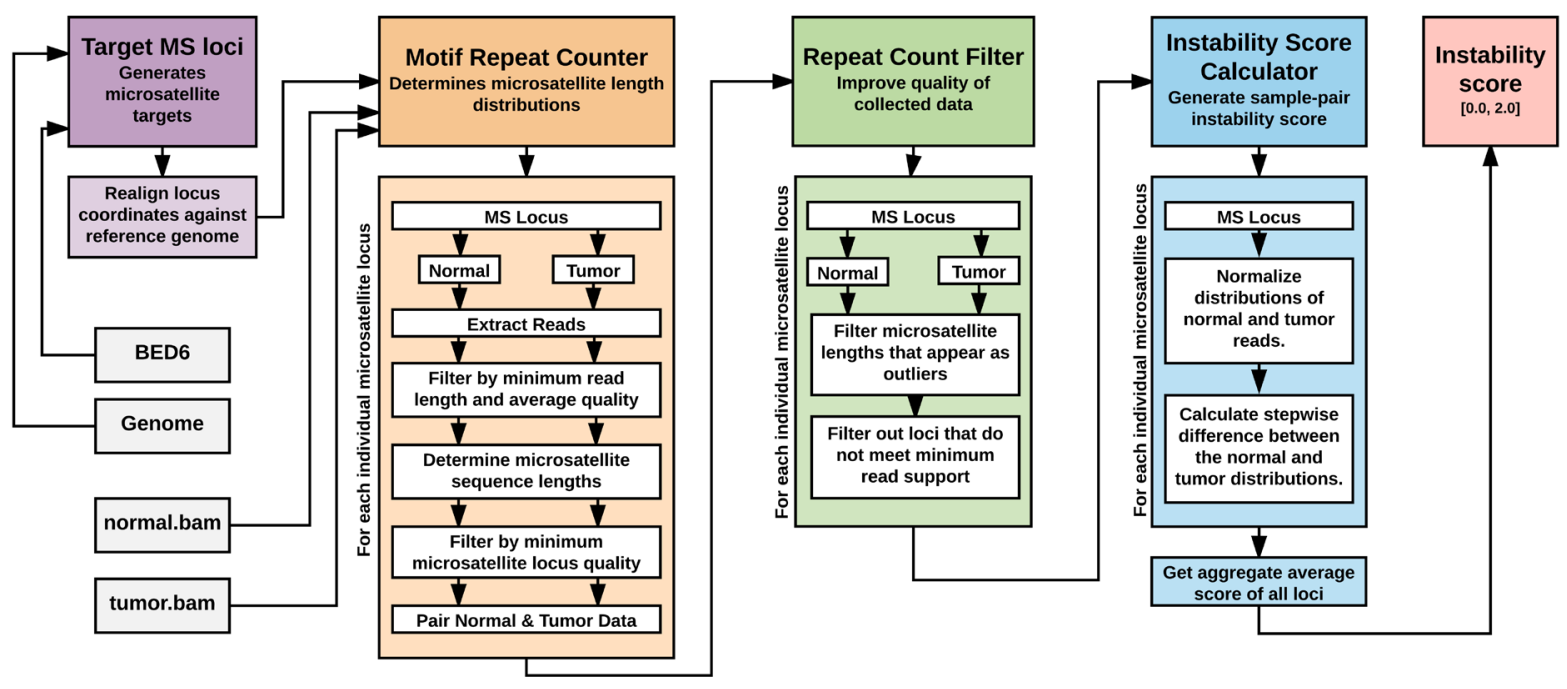

Figure 1: The schematic of the MANTIS analysis for MSI detection. Microsatellite loci are realigned against the reference genome to account for 0/1-based indexing differences. Per-locus microsatellite length distributions are determined from the normal and tumor BAM files by extracting locus-spanning reads; filtering out reads that fail to meet minimum length and average base quality requirements; determining the start position of the microsatellite motif within each read's sequence and the number of motif repeats; ensuring such reads meet minimum average locus quality and aren't prematurely truncated in the middle of a motif repeat. The generated normal and tumor length distributions are evaluated at each locus, with outlying length values (by default, $>3$ SD from mean) removed. Loci with substandard coverage are also removed. The support counts at each locus are then normalized separately for the normal and tumor sample and the stepwise difference between each distribution is calculated. Finally, the average of all difference scores is taken to generate the instability score for the normal-tumor sample pair. 
cohorts, tool performance was evaluated using stomach adenocarcinoma (STAD) sample pairs as a blinded test cohort (Figure 2). All three tools performed well with the STAD data, with MANTIS performing the best with $100 \%$ accuracy (Table 3), followed by MSISensor with 99\% accuracy and mSINGS with 96\% accuracy (Table 3 ).

To evaluate the extent that tool performance was affected by differences in sequencing, samples that had been sequenced at different sequencing centers with potentially different protocols and practices, were compared. For the comparison, we evaluated tool performance on a selection of MSI-H sample pairs that were each sequenced at two different centers (Supplemental Table S3). All three tools showed high concordance $\left(\mathrm{R}^{2}>0.99\right.$ in all cases) for the 20 UCEC tumor-normal pairs used in these comparisons. For the 4 COAD/READ pairs, MSISensor and MANTIS showed high concordance $\left(\mathrm{R}^{2}>0.99\right)$, however no concordance was observed with mSINGS $\left(\mathrm{R}^{2}=5.52 \cdot 10^{-7}\right)$. This indicated that while MANTIS and MSISensor are less affected due to their paired normal-tumor comparison model, mSINGS requires a statistically large enough population to generate a baseline from.

Finally, to assess the potential utility of MANTIS as well as other tools for pan-cancer MSI analysis, we tested MANTIS, mSINGS, and MSISensor with three additional cohorts of cancer: esophageal carcinoma (ESCA), uterine carcinosarcoma (UCS), and prostate adenocarcinoma (PRAD) (Supplemental Table S4). All three tools reached $100 \%$ accuracy with ESCA, and MSISensor and MANTIS were $100 \%$ accurate with UCS and PRAD. However, mSINGS only reached 50\% sensitivity (and 98.1\% accuracy) with UCS data, and had one false positive in
PRAD (98.3\% specificity and 98.3\% accuracy). Testing with these four additional disease cohorts further confirmed the accuracy of MANTIS, showing it may have superior pan-cancer applicability over the other two tools being compared.

\section{Computational performance}

Since resource limitations can affect the rate at which computational analysis of samples can be performed, we used the tumor and normal samples of TCGA-V5-A7RE (a known ESCA MSS case) for evaluating the computational performance of the three tools, as both deduplicated BAM files were close to 10 $\mathrm{GB}$ in size. We found that mSINGS performs considerably slower than MSISensor and MANTIS, with runtime at least five-fold longer (Supplemental Table S2). mSINGS also requires substantially more memory and disk space than both MSISensor and MANTIS. This is because over $99 \%$ of the 32 GB of disk space used was temporarily occupied by the mpileup file created by mSINGS with SAMtools as an intermediate step. MANTIS exhibited a $20.5 \%$ speed increase when run using three threads $v s$. one thread, and MSISensor had a $12.8 \%$ slowdown when using three threads (Supplemental Table S2). The lack of expected three-fold performance scaling with either tool may be due to testing in an I/O-bound computing environment. The lower resource consumption and faster performance of both MANTIS and MSISensor indicated they may be better suited than mSINGS for a resourcelimited environment, with MANTIS exhibiting the fastest runtimes.
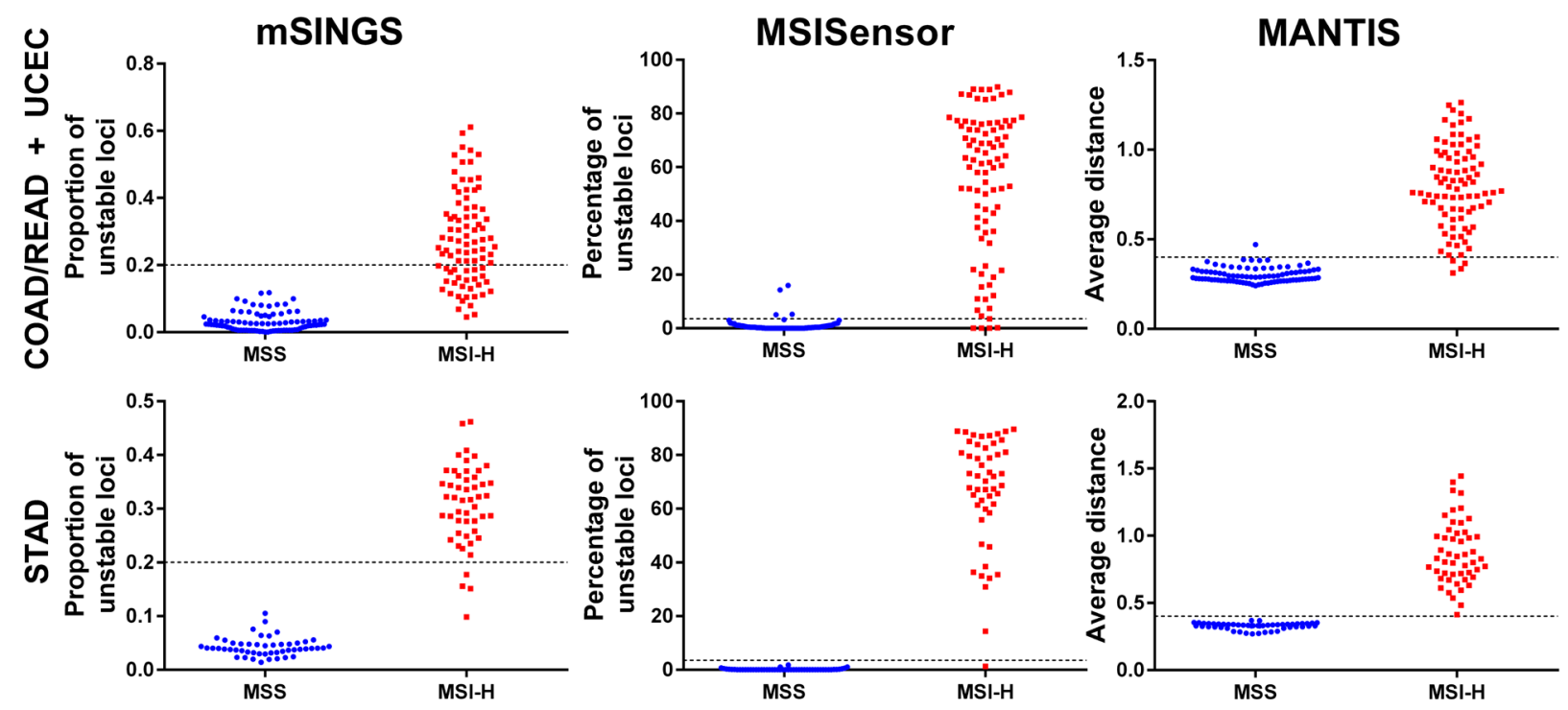

Figure 2: The cumulative distribution of MSI scores reported by mSINGS, MSISensor, and MANTIS for 275 COAD/ READ, UCEC and STAD tumor-normal data pairs. The dotted lines are the tools' respective thresholds to call a tumor MSI positive. 


\section{Accuracy of tools varies with both the number and specified loci evaluated}

To assess the effect of considering different numbers and selective microsatellite loci on MSI analysis, we identified the $10,20,30,40,50,100,250$, 500 and 1000 loci most predictive of a sample's status across COAD/READ, UCEC and STAD cohorts, for mSINGS, MSISensor and MANTIS (Supplemental File S5). Each tool was then run with its list of top loci from all three cancer types (Figure 3, Supplemental Figure S4). Notably, with its top 40 loci, mSINGS was more accurate than MSISensor and MANTIS with their top 40 loci $(98.2 \%, 91.8 \%$ and $97.4 \%$ accuracy, respectively) (Figure 3). mSINGS accuracy at 40 loci was higher with all three cancer types (Supplemental Table S5). In general, mSINGS performed better with fewer loci, MSISensor performed better with more loci, and MANTIS performed well consistently across a broader range of tested loci.

Previous studies have suggested that different MSI positive cancers may have specific microsatellite loci that are most commonly unstable $[10,11]$. For each MSI analysis tool, we sought to account for this by identifying the top-performing loci in each cancer type separately. Unlike in the analysis above, the $10,20,30,40,50,100$, 250,500 and 1000 best-performing loci for each tool were determined for each COAD/READ, UCEC and STAD cohort separately (Supplemental File S5). Each tool was then run over each cancer type, with respective top tool-specific and cancer-specific loci (Figure 4). Trends described in the previous analysis remained the same, with performance slightly higher throughout. However in UCEC at 40 loci, mSINGS performed better than
MSISensor and MANTIS (98.0\% accuracy, vs. 89.9\% and $94.9 \%$ respectively). Also, MANTIS and mSINGS both performed notably better (98.7\%) than MSISensor (83.6\%) when evaluating COAD/READ samples with 40 loci. The experiments show that the choice of loci being evaluated plays a part in tool performance. While an optimized target panel may allow all tools to perform well, MANTIS exhibits the most stable performance even without such optimizations, providing accurate performance using existing whole-exome data.

\section{DISCUSSION}

We have developed a new tool, MANTIS, for detecting MSI status using paired tumor-normal sequencing data. Unlike other tools, MANTIS analyzes the instability of a normal-tumor sample pair as an aggregate of loci instead of individual loci differences. The approach allows the tool to evaluate the general instability present in a tumor sample, using the data from the corresponding normal sample as an error-correcting baseline. Furthermore, by pooling the scores of all the loci and treating the average as the instability score, the evaluation benefits from the law of large numbers by reducing the impact that sequencing errors or poorly performing loci may have on the results.

We also analyzed the performance of MANTIS, mSINGS, and MSISensor with samples from six cancer types. Overall, MANTIS demonstrated high accuracy across a range of cancer types, and in many cases with restricted sets of well-performing loci. Prior tools have previously been applied to only one of two cancer types, endometrial (MSISensor) and colorectal (mSINGS). With their recommended thresholds, mSINGS and

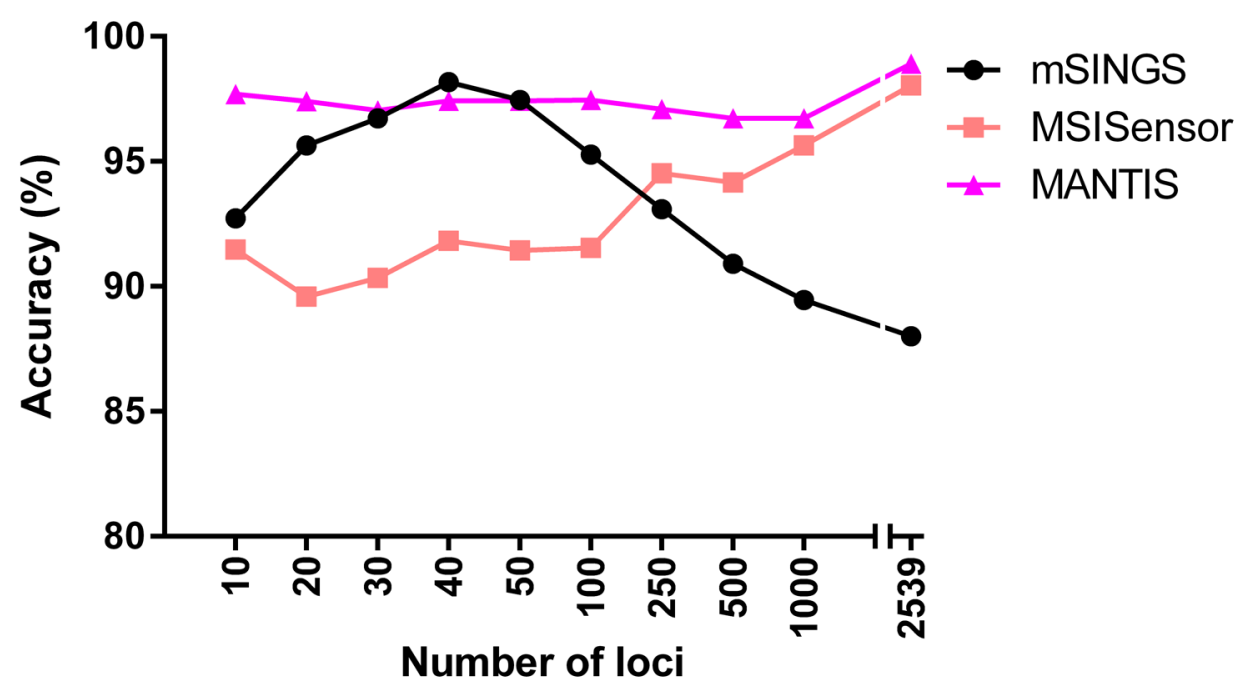

Figure 3: The performance of mSINGS, MSISensor and MANTIS with their respective top-performing loci. The performance of each tool in each COAD/READ, UCEC and STAD cohorts was evaluated with top tool-specific loci (10-1000). The results with 2539 loci (without loci shortlisting) are included for reference. 
MSISensor are less robust across a range of loci numbers than MANTIS. This appears to be due to the inclusion of poorly performing loci with low sensitivity in the full set of 2539 loci. mSINGS and MSISensor call loci unstable or stable, and MANTIS calculates the instability at each locus. Suboptimal loci may be missed entirely by mSINGS and MSISensor, but still have some increased instability in MSI-H vs. MSS samples. Niu et al. recommend a relatively low threshold of 3.5 for MSISensor ( $v s$.
$20 \%$ used by mSINGS and MSI-PCR). This seems to effectively compensate for these poorly performing loci with a large panel, but greatly reduces the specificity of MSISensor when these loci are removed, as in the lists of top-performing loci. Conversely, the threshold of 0.2 $(20 \%)$ used by mSINGS is effective with a smaller panel of well-performing loci such as the mSINGS authors' panel MSIplus [11], but this threshold limits the sensitivity of mSINGS with a larger panel (Table 3).
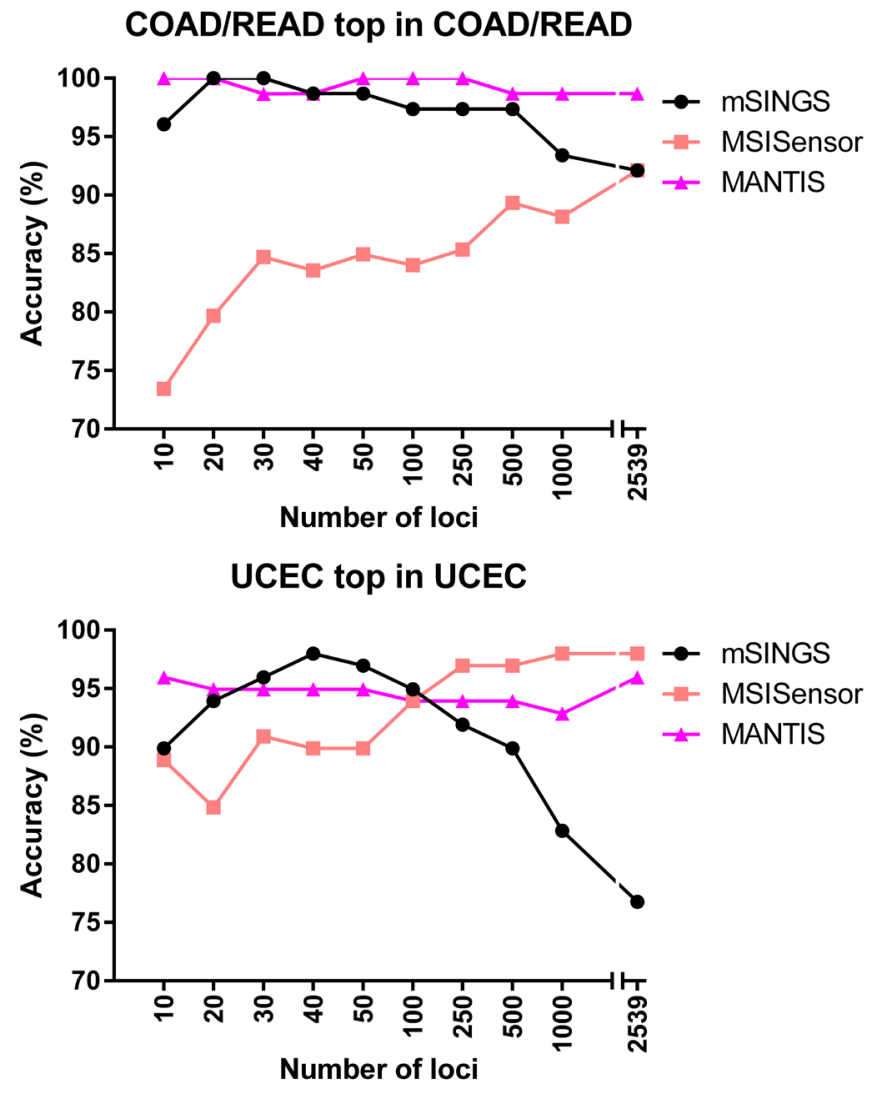

STAD top in STAD

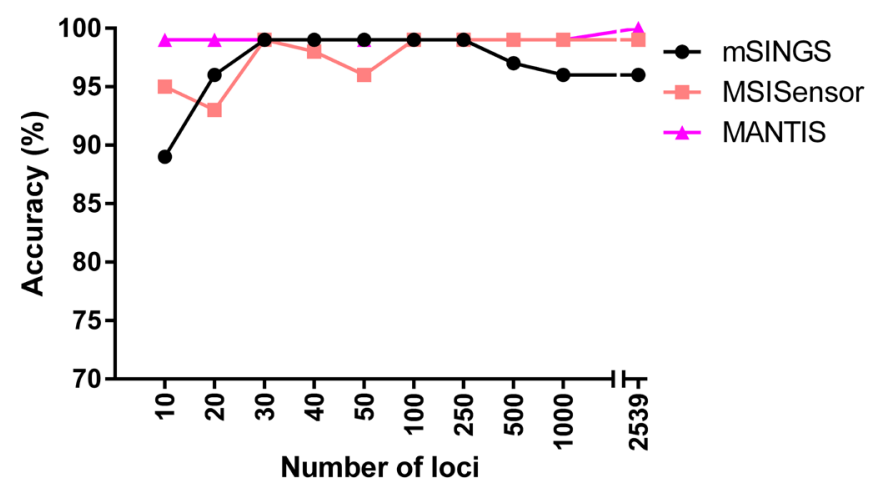

Figure 4: The performance of mSINGS, MSISensor and MANTIS with lists of loci top-performing in COAD/READ, UCEC or STAD. For each tool and for each cancer type (COAD/READ, UCEC or STAD), the top-performing loci were determined, and the performance of each tool in each cancer type was evaluated with lists of top loci (particular to only that cancer type) of varying length. The results with 2539 loci (without loci shortlisting) are included for reference. Results are broken down by cancer type. 
Results from UCEC were least concordant with the above trends. With the full set of 2539 loci, MSISensor performed best, followed closely by MANTIS and distantly by mSINGS. This may potentially arise from differences in the microsatellite instability signatures between COAD/READ and UCEC, the existence of which is supported by previous findings $[12,13]$. A recent overview of microsatellite instability across multiple cancer types by Hause et al. further found significant variance in the number of unstable loci present between samples from different diseases [14]. Another potential explanation is that, because mSINGS was developed only with COAD/READ data and MSISensor only with UCEC data, these tools may be overfit to those cancer types. MANTIS, in contrast, was developed and tested using data from multiple cancer types, alleviating the potential overfitting issues that may occur when only including data from a single disease.

Like any NGS-based method, MANTIS performance depends on read coverage, a limitation not shared by MSI-PCR and IHC. Most current clinical guidelines for management of MSI positive tumors are based on a percentage of unstable loci. Current approaches for MSI detection (such as mSINGS, MSISensor, and MSI-PCR) use a discrete fraction of unstable loci to determine to make calls on the status of a sample, but MANTIS provides a continuously valued MSI score that may provide greater utility in determining the level of MSI present in a tumor. Findings by Hause et al. give further credence to this hypothesis, indicating that microsatellite instability may best be viewed as a scaled genotype rather than a simple binary positive or negative classification [14].

We chose MANTIS, mSINGS, and MSISensor for this study since these three tools use NGS to directly assess microsatellite loci in DNA. Other NGS-based methods have been described that indirectly assess MSI through analysis of somatic mutations. MSIseq [15] employs machine learning classification techniques to correlate indels throughout repeat regions to MSI status. Stadler et al. [16] describe a method utilizing a custom NGS assay, and correlating somatic missense and nonsense mutations in protein-coding regions with MSI status. Additionally, Lu et al. [17] describe an algorithm for determining MSI status from RNA-seq data. MSIseq was not included in this study as it is a classifier that only reports MSI-H vs. non-MSI-H, without a score or percentage, or information about the instability of particular loci. The Stadler et al. and Lu et al. methods were not included since they cannot be run with the same whole exome sequencing input data, requiring a custom deep-sequenced panel and RNA-seq data, respectively.

Currently, MMR and MSI status are determined in the clinical setting with IHC and MSI-PCR. Conventional multiplex MSI-PCR testing is reported to have $97 \%$ sensitivity and $95 \%$ specificity [18]. IHC is reported to have $92.4 \%$ sensitivity and $99.6 \%$ specificity [36]. MSI-PCR with the standard five Bethesda loci is well described for COAD/READ and UCEC [4], but has been shown to perform less accurately in other diseases such as acute myeloid leukemia [10], and may miss MSI in other tumor types. Consideration of only five loci renders conventional MSI-PCR highly susceptible to errors in processing or interpretation of any one locus, and adding additional loci increases cost. IHC is able to effectively determine the presence or absence of the mismatch repair proteins targeted. Unfortunately, IHC cannot detect lossof-function mutations that do not affect the antigenicity of targeted proteins, or changes to MMR proteins not targeted [2]. Additionally, MSI-PCR and IHC require human interpretation, unlike computational NGS-based methods. Lastly, both MSI-PCR and IHC are clinical laboratory tests that consume fractions of patient tumor samples, unlike computational methods that could be multiplexed with other NGS assays for detecting somatic mutations.

As a tumor vs. normal algorithm, MANTIS avoids a time-consuming baseline generation step, eliminates potential baseline bias and allows processing of samples from different sequencing pipelines or tumor types without requiring a different baseline for each. Indeed, sequencing center bias may explain the discordance with mSINGS results from the $4 \mathrm{COAD} / \mathrm{READ}$ pairs sequenced at both BCM and BI (Supplemental Table S3). However, matched normal DNA is not always feasibly available for clinical laboratories that only sequence tumor samples, thus a tumor-only method such as mSINGS could reduce both sequencing time and cost to the patient.

The results of this study support several potential directions for future investigation. The accuracy of MANTIS with small numbers of loci suggests that MANTIS could be useful with a targeted sequencing panel designed for MSI testing in the clinic. We have shown that MANTIS performs well in six cancer types; however it (along with other MSI tools) should be further evaluated in a wider variety of cancers. Of particular interest would be evaluation of MANTIS in neurologic, hematologic, pediatric and other malignancies, in which the landscape of MSI is considerably less well described than with COAD/READ and UCEC. With further investigation, incorporating MANTIS into clinical NGS pipelines may permit MSI testing on a large scale, and improve access to emerging therapies that exploit microsatellite instability in cancer. 


\section{MATERIALS AND METHODS}

\section{MANTIS}

MANTIS is a tool for identifying microsatellite instability in paired tumor-normal patient samples (Figure 1). It is written as a Python program, utilizing the NumPy (developed with version 1.6.2) and Pysam (developed with version 0.8.3) libraries. Additionally, it requires the reference genome (developed with hg19) [10] in FASTA format to perform alignment of reads spanning the microsatellite loci. The matched normal and tumor inputs are required as indexed BAM files, aligned with any DNA sequence aligner. The targeted loci are required in a 6-column BED file, with the fourth column containing the motif of the microsatellite loci being targeted along with its repeat count in the reference genome, e.g. (AC) 12 . MANTIS includes a bundled $\mathrm{C}++$ program, RepeatFinder, used to identify microsatellite loci within a reference genome, and create the appropriate BED file. This BED file can be further filtered with BEDTools [20] for regions of interest. Multi-threading is supported and encouraged for larger samples, but is not necessary. More information about the parameters supported by MANTIS is included in the manual available with the software.

Targeted loci are first read from the provided BED file and realigned against the provided reference genome to account for differences between 0- and 1- based indexing. One locus at a time, the tool extracts overlapping reads from the tumor and normal BAM files and performs an initial quality control step to ensure the reads are of sufficient sequence length, meet a minimum average base quality score, and cover the entire targeted locus. Reads passing the initial filtering step are inspected individually to determine the starting position of the microsatellite motif within the read's sequence and the total number of repeats is determined by pattern matching the continuous motif pattern from that starting point. Once the repeat count is determined, a secondary quality control step takes place, ensuring that the locus was not truncated before reaching the end of the read's sequence, and that the microsatellite locus region has a sufficiently high average base quality score. The supporting read count for each of the repeat lengths is determined separately for the tumor and normal files to generate per-locus motif repeat count values.

Once the repeat counts are generated for each locus, a per-locus quality control step takes place. The repeat lengths for both the normal and tumor file are evaluated separately, with values too far from the mean (by default, beyond 3 standard deviations) discarded as outliers. After outliers are removed, each locus is checked for a total number of supporting reads to ensure there is sufficient support to generate a statistically significant distribution for both the normal and tumor files. Loci with substandard coverage are discarded.

The filtered locus repeat count data is then passed to the scoring algorithm that generates an instability score for the sample pair. First, each locus is evaluated separately, with the normal and tumor read distributions normalized to a fraction of each one's total reads, to account for any differences in sequencing depth and coverage. Then, absolute value of the stepwise difference between the tumor and normal distributions is determined:

$$
d=\sum_{r \in\left(R_{T} \cup R_{N}\right)}\left|T_{r}-N_{r}\right|
$$

Where $d=$ distance score, $R_{T}=$ repeat counts present in tumor, $R_{N}=$ repeat counts present in normal, $T_{r}=$ normalized read count in tumor supporting repeat of length $\mathrm{r}, N_{\mathrm{r}}=$ normalized read count in normal supporting repeat of length $r$. Once the scores for each locus are assigned, the average of all the locus instability scores is calculated, to provide a single numerical value representing the average aggregate instability present in the sample. Scores reported range from 0.0 (entirely stable) to 2.0 (entirely unstable). The MANTIS software and manual are freely available for download at https://github.com/OSU-SRLab/ MANTIS.

\section{Comparison of tools}

In addition to MANTIS, we tested mSINGS (commit \#2e00b6) by Salipante et al. [8] and MSISensor (version 0.2) by Niu et al. [9] (Table 1). mSINGS compares a tumor sample to a pooled normal baseline, generated from the distribution of unique alleles at each microsatellite locus in many normal samples. MSISensor, like MANTIS, compares a tumor sample with its matched normal sample. Both mSINGS and MSISensor determine the stability of each locus analyzed. mSINGS calls a locus unstable if the Z-score of the number of unique alleles at the locus relative to the baseline distribution exceeds a threshold (default: 2). MSISensor calls a locus unstable if a chi-square test of the repeat lengths in the tumor sample $v s$. the normal sample is statistically significant, after Benjamini correction [21]. Both tools then call the sample MSI-H or MSS if the percentage of unstable loci exceeds a threshold.

\section{Tool parameters}

mSINGS was run with Python 2.7.1, VarScan 2.3.6 $[22,23]$ and SAMtools 0.1.18 [24]. The wrapper script provided with mSINGS was modified (available upon request) to remove its dependency on SCons (http://scons. org/). We generated separate pooled normal baselines from all normal samples within a particular cancer type, according to the mSINGS documentation. The 
microsatellite loci-containing region $\mathrm{BED}$ and .intervals files packaged with mSINGS were used, which contained 2539 loci, as they are appropriate for whole-exome TCGA analyses according to the mSINGS documentation.

MSISensor was slightly modified to output sites that were not called with somatic microsatellite instability along with its other output files (patch files available upon request), and compiled from source. It was run over the test data with the following settings: single-threaded, minimal homopolymer size 1 , and minimal microsatellite size 1. All other options were left at their defaults. The microsatellite loci-containing region BED file packaged with mSINGS was used for fairness of comparison, as well as to restrict MSISensor to exomic regions.

MANTIS was run over the test data with three threads. The recommended quality settings for wholeexome data were used, as described in the included MANTIS manual. The microsatellite BED file was derived from the one provided by mSINGS.

\section{Target loci selection}

The 2539 target loci being analyzed were derived from the ones provided with mSINGS and captured in whole exome sequencing datasets, and used with all three tools. Most of the targeted loci (see Table 2) were monomer homopolymers of adenine or thymine $(95.08 \%)$, with only $4.04 \%$ of loci being repeats of dimers or longer polymers. This bias towards monomer repeats was expected since intronic mononucleotide repeats outnumber other repeat regions in the human genome [25].

\section{Sample data}

In this study, we used data from six cancer types: 76 colon and rectal adenocarcinomas (COAD/READ) [26], 99 uterine corpus endometrial carcinomas (UCEC) [27], 100 gastric adenocarcinomas (STAD) [28], 71 esophageal carcinomas (ESCA), 53 uterine carcinosarcomas (UCS) [29], and 59 prostate adenocarcinomas (PRAD) [30]. We define a "sample" as a single BAM and its accompanying BAI index file, for a tumor or a normal. We define a "pair" as two samples; a tumor and its matched normal, and define a "cohort" as all samples within a cancer type, for a total of 6 cohorts.

Data for all cohorts except PRAD were downloaded from the Cancer Genomics Hub (CGHub), using the CGHub-provided client GeneTorrent [31]. All samples were downloaded in the BAM format, pre-aligned to GRCh37/hg19 [19]. $76 \mathrm{COAD} / \mathrm{READ}$ pairs were downloaded, comprised of $38 \mathrm{MSI}-\mathrm{H}$ and $38 \mathrm{MSS}$. $\mathrm{COAD} / \mathrm{READ}$ data was sequenced at the Baylor College of Medicine (BCM) and the Washington University Genome Sequencing Center (WUGSC) (Supplemental Table S1B). 99 UCEC pairs were downloaded, comprised of $49 \mathrm{MSI}-\mathrm{H}$ and $50 \mathrm{MSS}$. All UCEC pairs were sequenced at WUGSC. Next, 100 STAD pairs were downloaded; 50 MSI-H and MSS. All STAD pairs were sequenced at the Broad Institute of MIT and Harvard (BI). The primary authors were blinded to the MSI status of these samples until after initial analysis with mSINGS, MSISensor and MANTIS was completed. Finally, 71 ESCA pairs were downloaded from TCGA; 2 MSI-H and $69 \mathrm{MSS}$, and 53 UCS pairs were downloaded; $2 \mathrm{MSI}-\mathrm{H}$ and $51 \mathrm{MSS}$. All ESCA and UCS pairs were sequenced at BI. PRAD data was downloaded from dbGaP (accession phs000915. v1.p1) [32] as FASTQ files, using the SRA toolkit [33]. All 59 available PRAD pairs were downloaded; 1 MSI positive and 58 MSI negative (according to the original study for which these samples were sequenced, which only differentiated between MSI positive and MSI negative). PRAD data was sequenced at BI and the University of Michigan (UM) (Supplemental Table S1C). Alignment to hg19 was performed with BWA 0.6.2 [34].

Each of the 916 BAM files (from the 458 tumornormal pairs in all six cancer types) were sorted and indexed with SAMtools 0.1.18. Deduplication was performed with Picard Tools 1.84 (http://broadinstitute. github.io/picard/), and facilitated with GNU Parallel [35]. The deduplicated BAM files were used for all downstream analyses. Samples used in these analyses are summarized in Supplemental Table S1A, Supplemental File S1.

\section{Tool performance evaluation}

mSINGS, MSISensor and MANTIS were first run on all 175 tumor-normal pairs from COAD/READ and UCEC (Supplemental File S2). A threshold was used for each tool, above which a tumor-normal pair is called MSI positive. For mSINGS, 0.2 (20\% of loci called unstable) was used as the threshold for differentiation of MSI positive from MSS predictions, as this is consistent with both MSI-PCR scoring and the threshold used by Salipante et al. A threshold of 3.5\% (of loci called unstable) was used for MSISensor, as recommended by Niu et al. For MANTIS, a threshold of 0.4 (average of loci difference scores $>0.4$ ) performed best in testing (see: Results; Relative tool performance), and was used for other analyses. For each tool, the number of true positives, false positives, true negatives, and false negatives was calculated with respect to MSI-PCR status as a gold standard, and this was used to calculate the sensitivity, specificity, error rate, and accuracy of each tool both overall and within each cancer type. Error rate was calculated as (incorrect calls / total calls), and accuracy as ( 1 - error rate $=$ correct calls $/$ total calls $)$. Note that error rate and accuracy depend on the samples being tested, and cannot be generalized to other data sets, as can sensitivity and specificity. 95\% confidence intervals for sensitivity and specificity were calculated using the Wilson score interval with continuity correction [36]. In addition, ranges 
of thresholds were tested for all three tools in order to determine which thresholds provided optimal performance with the COAD/READ and UCEC test data, allowing comparison of best-case performance. 300 thresholds ranging from 0.001 to 0.3 were tested for mSINGS, 400 thresholds ranging from $0.1 \%$ to $40 \%$ were tested for MSISensor, and 600 thresholds ranging from 0.001 to 0.6 were tested for MANTIS. After a threshold of 0.4 was chosen for MANTIS, we ran mSINGS, MSISensor and MANTIS over the 100 STAD, 71 ESCA, 53 UCS and 59 PRAD tumor-normal pairs. Tool parameters were selected and performance analyses were performed as described earlier.

For each tool, we measured potential bias arising from differences in sequencing and alignment protocols at different sequencing centers. In addition to the sample data used for tool performance comparison, TCGA data from 4 tumor-normal COAD/READ MSI-H pairs sequenced at both $\mathrm{BI}$ and $\mathrm{BCM}$, as well as from 20 tumor-normal UCEC MSI-H pairs sequenced at both BI and WUGSC, was downloaded and preprocessed as earlier (see: Sample data) (Supplemental File S3). After deduplication, these pairs were analyzed with mSINGS, MSISensor and MANTIS. MSISensor and MANTIS were run with the parameters described earlier (see: Tool parameters). For mSINGS, the normal BAM files were used to generate separate baselines for each cancer type and sequencing center.

In addition to evaluating the statistical performance of each tool, we also evaluated their computational performance (Supplemental Table S2). All three tools were profiled with the runtime and memory usage metrics supplied by the PBS (Portable Batch System) cluster queueing system. MSISensor and MANTIS were tested with one and three threads to evaluate changes in performance (mSINGS does not support multithreading).

\section{Loci number comparison}

For each of the three tools tested, the top-performing $10,20,30,40,50,100,250,500$ and 1000 microsatellite loci within the COAD/READ, UCEC and STAD samples were determined, individually within each cancer type and across all three cohorts. ESCA, UCS and PRAD were not included in this as only five MSI-H cases were available from these cancer types. To determine the top $n$ loci for mSINGS and MSISensor, the accuracy of each locus was calculated as if only that locus were to be used to call the MSI status of each tumor sample. For MANTIS, since it calculates instability scores instead of assigning per-locus stability statuses, a difference of averages was calculated for each locus, defined as:

$$
a=\frac{\sum_{i \in H} d_{i}}{|H|}-\frac{\sum_{i \in S} d_{i}}{|S|}
$$

Where $a=$ difference of averages, $H=$ set of MSI-H samples that cover the locus, $S=$ set of MSS samples that cover the locus, and $d_{i}=$ distance score of the locus in sample $i$. To compensate for varying locus coverage across samples, this score (accuracy or difference of averages) was then multiplied by the square of the proportion of pairs that had sufficient read coverage at that locus to consider it, as follows:

$$
l=a c^{2}
$$

Where $l=$ locus score, $a=$ accuracy or difference of averages, and $c=$ proportion of samples that cover the locus. This yielded a performance score for each locus, which allowed all 2539 loci to be ranked. The sensitivity, specificity, error rate, and accuracy of each tool with each loci list was then calculated, both for all three of these cancer types and overall (Supplemental File S4).

\section{Computing resources}

Alignment, deduplication, MSI calling with all three tools, and performance calculations were performed on the Oakley supercomputer at the Ohio Supercomputer Center (https://www.osc.edu/supercomputing/computing/oakley). Figures were generated using GraphPad Prism (version 6.07) and Microsoft ${ }^{\circledR}$ Excel $^{\mathrm{TM}}$ 2010. All other performance calculations were performed with custom Perl scripts and Excel $^{\mathrm{TM}}$.

\section{ACKNOWLEDGMENTS}

We would like to acknowledge Pelotonia, the Prostate Cancer Foundation, the American Lung Association, the American Cancer Society, The Cancer Genome Atlas, Stand Up To Cancer, the Ohio Supercomputer Center (OSC), and the Comprehensive Cancer Center (CCC) at the Ohio State University Wexner Medical Center. The results published here are in part based upon data generated by the TCGA Research Network. We are grateful for administrative support from Jenny Badillo.

\section{FUNDING}

This work was supported by the American Cancer Society [grant number MRSG-12-194-01-TBG]; the Prostate Cancer Foundation; the National Human Genome Research Institute [grant number UM1HG006508]; the National Cancer Institute [grant number UH2CA202971]; the American Lung Association; Pelotonia; and Fore Cancer Research. Funding for open access charge: American Cancer Society. 


\section{Editorial note}

This paper has been accepted based in part on peerreview conducted by another journal and the authors' response and revisions as well as expedited peer-review in Oncotarget.

\section{REFERENCES}

1. Kelkar YD, Strubczewski N, Hile SE, Chiaromonte F, Eckert KA and Makova KD. What is a microsatellite: a computational and experimental definition based upon repeat mutational behavior at $\mathrm{A} / \mathrm{T}$ and $\mathrm{GT} / \mathrm{AC}$ repeats. Genome Biol Evol. 2010; 2:620-635.

2. Shia J. Evolving approach and clinical significance of detecting DNA mismatch repair deficiency in colorectal carcinoma. Semin Diagn Pathol. 2015; 32(5):352-361.

3. Aaltonen LA, Peltomäki P, Leach FS, Sistonen P, Pylkkänen L, Mecklin JP, Järvinen H, Powell SM, Jen J and Hamilton SR. Clues to the pathogenesis of familial colorectal cancer. Science. 1993; 260(5109):812-816.

4. Armaghany T, Wilson JD, Chu Q and Mills G. Genetic alterations in colorectal cancer. Gastrointest Cancer Res. 2012; 5(1):19-27.

5. Dudley JC, Lin MT, Le DT and Eshleman JR. Microsatellite Instability as a Biomarker for PD-1 Blockade. Clin Cancer Res. 2016; 22(4):813-820.

6. Le DT, Uram JN, Wang H, Bartlett BR, Kemberling $\mathrm{H}$, Eyring AD, Skora AD, Luber BS, Azad NS, Laheru D, Biedrzycki B, Donehower RC, Zaheer A, et al. PD-1 Blockade in Tumors with Mismatch-Repair Deficiency. N Engl J Med. 2015; 372(26):2509-2520.

7. Boland CR, Thibodeau SN, Hamilton SR, Sidransky D, Eshleman JR, Burt RW, Meltzer SJ, Rodriguez-Bigas MA, Fodde R, Ranzani GN and Srivastava S. A National Cancer Institute Workshop on Microsatellite Instability for cancer detection and familial predisposition: development of international criteria for the determination of microsatellite instability in colorectal cancer. Cancer Res. 1998; 58(22):5248-5257.

8. Salipante SJ, Scroggins SM, Hampel HL, Turner EH and Pritchard CC. Microsatellite instability detection by next generation sequencing. Clin Chem. 2014; 60(9):1192-1199.

9. Niu B, Ye K, Zhang Q, Lu C, Xie M, McLellan MD, Wendl $\mathrm{MC}$ and Ding L. MSIsensor: microsatellite instability detection using paired tumor-normal sequence data. Bioinformatics. 2014; 30(7):1015-1016.

10. Faulkner RD, Seedhouse CH, Das-Gupta EP and Russell NH. BAT-25 and BAT-26, two mononucleotide microsatellites, are not sensitive markers of microsatellite instability in acute myeloid leukaemia. Br J Haematol. 2004; 124(2):160-165.

11. Hempelmann JA, Scroggins SM, Pritchard CC and
Salipante SJ. MSIplus for Integrated Colorectal Cancer Molecular Testing by Next-Generation Sequencing. J Mol Diagn. 2015; 17(6):705-714.

12. Alhopuro P, Sammalkorpi H, Niittymäki I, Biström $M$, Raitila A, Saharinen J, Nousiainen K, Lehtonen HJ, Heliövaara E, Puhakka J, Tuupanen S, Sousa S, Seruca $\mathrm{R}$, et al. Candidate driver genes in microsatellite-unstable colorectal cancer. Int J Cancer. 2012; 130(7):1558-1566.

13. Kim TM, Laird PW and Park PJ. The landscape of microsatellite instability in colorectal and endometrial cancer genomes. Cell. 2013; 155(4):858-868.

14. Hause RJ, Pritchard CC, Shendure J and Salipante SJ. Classification and characterization of microsatellite instability across 18 cancer types. Nat Med. 2016; 22(11):1342-1350.

15. Huang MN, McPherson JR, Cutcutache I, Teh BT, Tan P and Rozen SG. MSIseq: Software for Assessing Microsatellite Instability from Catalogs of Somatic Mutations. Sci Rep. 2015; 5:13321.

16. Stadler ZK, Battaglin F, Middha S, Hechtman JF, Tran C, Cercek A, Yaeger R, Segal NH, Varghese AM, ReidyLagunes DL, Kemeny NE, Salo-Mullen EE, Ashraf A, et al. Reliable Detection of Mismatch Repair Deficiency in Colorectal Cancers Using Mutational Load in NextGeneration Sequencing Panels. J Clin Oncol. 2016; 34(18):2141-2147.

17. Lu Y, Soong TD and Elemento O. A novel approach for characterizing microsatellite instability in cancer cells. PLoS One. 2013; 8(5):e63056.

18. Zhang $\mathrm{X}$ and $\mathrm{Li}$ J. Era of universal testing of microsatellite instability in colorectal cancer. World J Gastrointest Oncol. 2013; 5(2):12-19.

19. Lander ES, Linton LM, Birren B, Nusbaum C, Zody MC, Baldwin J, Devon K, Dewar K, Doyle M, FitzHugh W, Funke R, Gage D, Harris K, et al. Initial sequencing and analysis of the human genome. Nature. 2001; 409(6822):860-921.

20. Quinlan AR and Hall IM. BEDTools: a flexible suite of utilities for comparing genomic features. Bioinformatics. 2010; 26(6):841-842.

21. Benjamini $\mathrm{Y}$ and Hochberg Y. Controlling the False Discovery Rate: A Practical and Powerful Approach to Multiple Testing. Journal of the Royal Statistical Society Series B (Methodological). 1995; 57(1):289-300.

22. Koboldt DC, Chen K, Wylie T, Larson DE, McLellan MD, Mardis ER, Weinstock GM, Wilson RK and Ding L. VarScan: variant detection in massively parallel sequencing of individual and pooled samples. Bioinformatics. 2009; 25(17):2283-2285.

23. Koboldt DC, Zhang Q, Larson DE, Shen D, McLellan MD, Lin L, Miller CA, Mardis ER, Ding L and Wilson RK. VarScan 2: somatic mutation and copy number alteration discovery in cancer by exome sequencing. Genome Res. 2012; 22(3):568-576. 
24. Li H, Handsaker B, Wysoker A, Fennell T, Ruan J, Homer N, Marth G, Abecasis G, Durbin R and Subgroup GPDP. The Sequence Alignment/Map format and SAMtools. Bioinformatics. 2009; 25(16):2078-2079.

25. Tóth G, Gáspári Z and Jurka J. Microsatellites in different eukaryotic genomes: survey and analysis. Genome Res. 2000; 10(7):967-981.

26. Network TCGAR. Comprehensive molecular characterization of human colon and rectal cancer. Nature. 2012; 487(7407):330-337.

27. Network TCGAR. Integrated genomic characterization of endometrial carcinoma. Nature. 2013; 497(7447):67-73.

28. The Cancer Genome Atlas Research N. Comprehensive molecular characterization of gastric adenocarcinoma. Nature. 2014; 513(7517):202-209.

29. The Cancer Genome Atlas Research N, Weinstein JN, Collisson EA, Mills GB, Shaw KRM, Ozenberger BA, Ellrott K, Shmulevich I, Sander C and Stuart JM. The Cancer Genome Atlas Pan-Cancer analysis project. Nat Genet. 2013; 45(10):1113-1120.

30. Robinson D, Van Allen EM, Wu YM, Schultz N, Lonigro RJ, Mosquera JM, Montgomery B, Taplin ME, Pritchard CC, Attard G, Beltran H, Abida W, Bradley RK, et al. Integrative clinical genomics of advanced prostate cancer. Cell. 2015; 161(5):1215-1228.
31. Wilks C, Cline MS, Weiler E, Diehkans M, Craft B, Martin C, Murphy D, Pierce H, Black J, Nelson D, Litzinger B, Hatton T, Maltbie L, et al. The Cancer Genomics Hub (CGHub): overcoming cancer through the power of torrential data. Database (Oxford). 2014; 2014.

32. Mailman MD, Feolo M, Jin Y, Kimura M, Tryka K, Bagoutdinov R, Hao L, Kiang A, Paschall J, Phan L, Popova N, Pretel S, Ziyabari L, et al. The NCBI dbGaP database of genotypes and phenotypes. Nat Genet. 2007; 39(10):1181-1186.

33. Leinonen R, Sugawara H, Shumway M and Collaboration INSD. The sequence read archive. Nucleic Acids Res. 2011; 39(Database issue):D19-21.

34. Li H and Durbin R. Fast and accurate short read alignment with Burrows-Wheeler transform. Bioinformatics. 2009; 25(14):1754-1760.

35. Tange O. (2011). GNU Parallel - The Command-Line Power Tool. ;login: The USENIX Magazine, pp. 42-47.

36. Newcombe RG. Two-sided confidence intervals for the single proportion: comparison of seven methods. Stat Med. 1998; 17(8):857-872. 